\title{
Destete hiper-precoz y crecimiento post-destete de gazapos de chinchilla (Chinchilla lanigera)
}

\author{
Nistal, A.J. ${ }^{1,3}$; Mazufero, K. ${ }^{3}$; Zapata, M.D. ${ }^{1,3}$; Di Masso, R.J. ${ }^{2,4}$
}

Cátedras de ${ }^{1}$ Fisiología y ${ }^{2}$ Genética, Facultad de Ciencias Veterinarias, Universidad Nacional de Rosario (UNR). ${ }^{3}$ Módulo de producción de chinchillas, Escuela Agrotécnica Lib.Gral.San Martín, UNR. ${ }^{4} \mathrm{CIC}-\mathrm{UNR}$, Ovidio Lagos y Ruta 33, Casilda 2170, Santa Fé, Argentina. E-mail: anistal@fveter.unr.edu.ar

\begin{abstract}
Resumen
Nistal, A.J.; Mazufero, K.; Zapata, M.D.; Di Masso, R.J.: Destete hiper-precoz y crecimiento post-destete de gazapos de chinchilla (Chinchilla lanigera). Rev vet 29(1): 31-34, 2018. En la chinchilla la duración promedio de la lactancia es de 60 días. La evidencia experimental disponible acerca de la factibilidad de acortarla indica que ni el destete anticipado (42 días) ni el destete precoz (32 días) afectan negativamente el crecimiento post-destete de los gazapos. El objetivo de este trabajo fue evaluar el efecto del destete hiper-precoz ( 25 días de edad) sobre la ganancia diaria de peso de los gazapos en el post-destete inmediato, en función de su peso corporal previo a la separación de la madre, con el fin de determinar la factibilidad de extremar aún más esta estrategia de manejo. Se evaluaron en total 21 gazapos pertenecientes a camadas de dos individuos, los que fueron separados de sus madres a los 25 días de edad. Los gazapos se discriminaron en tres categorías de acuerdo a su peso individual al destete: gazapos con pesos corporales menores al percentil 33 (grupo liviano); gazapos con pesos comprendidos entre los percentiles 33 y 66 (grupo intermedio) y gazapos con pesos corporales mayores al percentil 66 (grupo pesado). Los resultados indican que, independientemente del peso del gazapo al momento de ser separado de su madre, el destete a los 25 días de vida es una práctica factible de ser aplicada en los criaderos comerciales de chinchillas en tanto la misma no repercute negativamente sobre el crecimiento posterior de las crías a la vez que cumple con el objetivo de permitir que la hembra interrumpa su anestro lactacional y se reincorpore antes al ciclo reproductivo, contribuyendo de esta manera a disminuir el período inter-parto.
\end{abstract}

Palabras clave: Chinchilla lanigera, peso corporal, crecimiento pre- y post-destete, lactancia, reducción del anestro lactacional.

\begin{abstract}
Nistal, A.J.; Mazufero, K.; Zapata, M.D.; Di Masso, R.J.: Hyper-precocious weaning and post-weaning growth in Chinchilla lanigera pups. Rev vet 29(1): 31-34, 2018. In chinchilla the average duration of lactation is 60 days. Available experimental evidence on the feasibility of its shortening indicates that neither anticipated weaning (42 days) nor early weaning (32 days) negatively affect the post-weaning growth of the offspring. The objective of this study was to evaluate the effect of hyper-precocious weaning ( 25 days of age) on the daily weight gain of the offspring in the immediate post-weaning period, according to their body weight prior to the separation of the mother, in order to determine the feasibility of further maximizing this management strategy. A total of 21 pups belonging to litters of two individuals, were evaluated. Pups were separated from their mothers by 25 days of age and divided into three categories according to their individual weaning weight: those with body weights below the 33rd percentile (light group); those with weights included between the percentiles 33 and 66 (intermediate group) and those with body weights upper the percentile 66 (heavy group). The results indicate that, regardless of the weight of the pup when separated from its mother, weaning at 25 days of age is a practice feasible to be applied in chinchilla commercial breeding systems, as it does not affect negatively the subsequent growth of the offspring. At the same time, this practice fulfills the objective of allowing the female to interrupt her lactational anestrus and so resume before to the reproductive cycle, thereby contributing to a decrease in the inter-partum period.
\end{abstract}

Key words: Chinchilla lanigera, body weight, pre-weaning and post-weaning growth, lactation, reduction lactational anestrus. 


\section{INTRODUCCIÓN}

La cría comercial de chinchillas en cautiverio se organiza en base a familias reproductoras integradas -habitualmente- por un macho y seis hembras. El alojamiento utilizado consiste en una batería de jaulas provista con compartimentos individuales para cada hembra, en la que el macho carece de habitáculo propio y deambula por un pasillo o corredor con troneras que permiten el acceso a las reproductoras de su familia.

Para evitar que las hembras utilicen la tronera para salir de su jaula e introducirse en la de otra hembra con las consiguientes peleas, se les coloca un collar metálico. Las troneras de la batería de cada familia en reproducción se mantienen abiertas en forma permanente mientras las hembras no se encuentran con cría, incluso cuando están gestando, en tanto el macho sólo tiene acceso al agua y al alimento si puede introducirse en alguna de las jaulas de su harén.

Una vez ocurrido el parto la tronera permanece abierta durante las $72 \mathrm{~h}$ inmediatas posteriores, de manera tal de aprovechar la presentación de un eventual celo posparto fértil, común en esta especie ${ }^{6,8,9}$ y luego se cierra hasta el momento del destete para evitar que los gazapos utilicen el pasillo del macho para acceder a las jaulas de otras hembras, las que -por lo general- se muestran muy agresivas con las crías que no son propias.

En cuanto a la duración de la lactancia, la bibliografía menciona valores promedio de dos meses $2,3,5$, 7,9 con tendencia a acortarla ${ }^{4}$ respetando un período mínimo de amamantamiento de 25 días ${ }^{8}$ para asegurar la sobrevida de las crías.

En los sistemas productivos de chinchillas, la prolongación de la lactancia suele implementarse en ocasiones como una práctica tendiente a favorecer el crecimiento de las crías cuando se observa que éstas no presentan un desarrollo adecuado ${ }^{1}$, si bien en la actualidad se tiende a anticipar el destete, separando a los gazapos a una edad más temprana, con el objeto de reincorporar a las hembras al ciclo reproductivo anticipando el acceso del macho a las mismas ${ }^{4}$.

Acortar la duración de la lactancia requiere disponer de información acerca del potencial impacto que dicha práctica puede producir sobre los gazapos. En estudios realizados en esta especie sobre destete anticipado (42 días) y destete precoz (32 días) se demostró (Nistal \& Di Masso 2015) que ambas prácticas de manejo no afectan negativamente el crecimiento post-destete de los gazapos.

El objetivo de este trabajo fue evaluar el efecto del destete hiper-precoz ( 25 días de edad) sobre la ganancia diaria de peso de los gazapos en el post-destete inmediato, en función de su peso corporal previo a la separación de la madre, con el fin de determinar la factibilidad de extremar aún más esta estrategia de manejo.

\section{MATERIAL Y MÉTODOS}

Se evaluaron en total 21 gazapos pertenecientes a camadas de dos individuos, nacidos y criados en el
Módulo-Didáctico Productivo de la Escuela Agrotécnica Libertador General San Martín de la Universidad de Rosario, Argentina, los que fueron separados de sus madres a los 25 días de edad.

Los gazapos se discriminaron en tres categorías de acuerdo a su peso individual al destete: gazapos con pesos corporales menores al percentil 33 (grupo liviano GL); gazapos con pesos comprendidos entre los percentiles 33 y 66 (grupo intermedio GI) y gazapos con pesos corporales mayores al percentil 66 (grupo pesado GP).

La efectividad de la discriminación de los gazapos en grupos de pesos significativamente diferentes al destete, se constató con un análisis de la variancia a un criterio de clasificación seguido de la prueba de comparaciones múltiples de Bonferroni.

Para cada gazapo, en cada uno de los tres grupos, se calculó la ganancia diaria de peso en tres períodos sucesivos: en la semana previa al destete (PreD: 18-25 días de edad), en la primera semana posterior al destete (PosD1: 26-32 días de edad) y en la segunda semana posterior al destete (PosD2: 33-39 días de edad).

Los datos entre períodos tomados de a dos se compararon con una prueba $t$ de Student para datos apareados utilizando al gazapo como criterio de apareamiento.

El efecto del grupo de pertenencia sobre la ganancia diaria de peso corporal de los gazapos en cada uno de los tres períodos considerados, se evaluó con un análisis de la variancia a un criterio de clasificación, seguido de la prueba de comparaciones múltiples de Bonferroni.

\section{RESULTADOS}

La Tabla 1 resume los pesos al destete de los gazapos según su grupo de pertenencia. La diferencia en el peso corporal promedio de los tres grupos fue estadísticamente significativa $(\mathrm{p}<0,001)$.

La Tabla 2 presenta los valores de las ganancias diarias de peso de los gazapos de cada uno de los tres grupos de peso, en la semana previa al destete y en las dos semanas posteriores al mismo.

La ganancia diaria de peso pre-destete de los gazapos del grupo liviano no difirió significativamente de la observada en los dos períodos del post-destete, como tampoco presentaron diferencia los aumentos de peso corporal observados en ambos períodos del postdestete.

Tabla 1. Peso corporal de gazapos de chinchilla destetados a los 25 días de edad, discriminados en tres grupos (liviano, intermedio y pesado).

\begin{tabular}{lccc}
\hline peso $(\mathrm{g})$ & $\mathrm{GL}$ & $\mathrm{GI}$ & $\mathrm{GP}$ \\
\hline $\mathrm{n}$ & 7 & 7 & 7 \\
mínimo & 117 & 128 & 147 \\
máximo & 124 & 144 & 166 \\
promedio & 122,4 & 136,9 & 157,9 \\
error estándar & 0,972 & 1,993 & 2,939 \\
\hline
\end{tabular}


Tabla 2. Ganancias medias diarias de peso (g/día) pre-destete y postdestete de gazapos de chinchilla con destete hiper-precoz (25 días) discriminados por su peso al destete.

\begin{tabular}{lcccccc}
\hline \multirow{3}{*}{ grupo } & \multicolumn{3}{c}{ período } & & contraste \\
\cline { 2 - 7 } & PreD & PosD1 & PosD2 & PreD vs & PreD vs & PosD1 vs \\
& $18-25$ días & $25-32$ días & $32-39$ días & PosD1 & PosD2 & PosD2 \\
\hline \multirow{2}{*}{ GL } & 3,10 & 3,72 & 4,34 & $\mathrm{t}=0,719$ & $\mathrm{t}=1,443$ & $\mathrm{t}=1,158$ \\
& $\pm 0,761$ & $\pm 0,713$ & $\pm 0,402$ & $\mathrm{p}=0,499$ & $\mathrm{p}=0,199$ & $\mathrm{p}=0,291$ \\
\multirow{2}{*}{ GI } & 3,41 & 2,71 & 5,09 & $\mathrm{t}=0,846$ & $\mathrm{t}=2,888$ & $\mathrm{t}=3,235$ \\
& $\pm 0,413$ & $\pm 0,652$ & $\pm 0,411$ & $\mathrm{p}=0,430$ & $\mathrm{p}=0,028$ & $\mathrm{p}=0,018$ \\
\multirow{2}{*}{ GP } & 5,05 & 2,98 & 5,76 & $\mathrm{t}=2,271$ & $\mathrm{t}=1,132$ & $\mathrm{t}=4,242$ \\
& $\pm 0,499$ & $\pm 0,620$ & $\pm 0,275$ & $\mathrm{p}=0,029$ & $\mathrm{p}=0,295$ & $\mathrm{p}=0,005$ \\
\hline
\end{tabular}

Tamaño muestral $=7$ gazapos por grupo. Todos los valores corresponden a la media aritmética \pm error estándar. $\mathrm{t}=$ estadística $\mathrm{t}$ de Student; $\mathrm{p}$ : probabilidad asociada.

Por su parte, los gazapos pertenecientes al grupo intermedio no mostraron diferencias en la ganancia diaria de peso entre la semana previa al destete (PreD) y la semana posterior al mismo (PosD1), pero sí entre el primero de dichos períodos y el correspondiente a la segunda semana post-destete, y entre este último y el valor registrado en la primera semana posterior al destete, siendo dicha ganancia de peso mayor en la segunda semana posterior a la separación de la madre.

Por último, en los gazapos del grupo pesado, la ganancia diaria de peso en la semana previa a la separación de sus madres fue significativamente mayor a la observada en la primera semana posterior a dicho evento, sin diferenciarse significativamente de la registrada en la segunda semana de vida independiente. La ganancia de peso diario de estos gazapos en la segunda semana de vida independiente, fue significativamente mayor a la registrada en la primera semana posterior al destete.

La comparación de las ganancias diarias de peso de los tres grupos de gazapos, en cada uno de los tres períodos, puso en evidencia la ausencia de diferencias estadísticamente significativas en la semana previa al destete $(p=0,085)$ y en la primera semana posterior al mismo $(p=0,638)$, mientras que en la segunda semana post-destete se observaron diferencias entre el grupo liviano y el grupo pesado, presentando este último un mayor aumento diario $(p=0,045)$, sin diferencias estadísticamente significativas entre los grupos liviano e intermedio, ni entre este último y el grupo pesado.

\section{DISCUSIÓN}

El destete hiper-precoz, entendiendo por tal aquel efectuado a los 25 días de edad, mejoró el desempeño de los gazapos del grupo liviano en términos de crecimiento dimensional. El cambio de ambiente no generó en ellos detrimento alguno en su velocidad de crecimiento, sino que, por el contrario, se produjo un aumento en la ganancia diaria de peso, ya evidente en la primera semana, del $20 \%$ respecto de la registrada en la última semana de lactancia.

Esta diferencia aumentó al $40 \%$ en la segunda semana de vida independiente sobre el valor observado durante la última semana previa al destete. Para este grupo de animales de bajo peso corporal, la prolongación de la lactancia no representaría una ventaja si se utiliza como indicador la ganancia diaria de peso. En el caso de los grupos intermedio y pesado, el destete a los 25 días dio lugar a un comportamiento similar.

En ambos se produjo una disminución de la velocidad de crecimiento en la primera semana de vida independiente y una recuperación de la misma durante la segunda semana post-destete, que superó los valores registrados previamente a la separación. En los gazapos pertenecientes al grupo intermedio, la ganancia diaria de peso disminuyó un $20 \%$ cuando se compara el valor del pre-destete con el de la primera semana posterior al destete, disminución que se duplicó en el caso de los gazapos del grupo pesado poniendo en evidencia un mayor efecto detrimental de la práctica en estos últimos.

En la segunda semana de vida independiente se produjo un aumento significativo en la ganancia diaria de peso con respecto a la observada en la primera semana, llegando a superar -en el caso de los gazapos del grupo intermedio- la velocidad de crecimiento observada en el pre-destete.

Cuando se comparan las ganancias de peso de cada grupo, en la semana pre-destete se observa la tendencia esperada con valores mínimos para los gazapos del grupo liviano, seguidos por los del grupo intermedio y valores máximos para los del grupo pesado. En la primera semana posterior al destete los distintos grupos no se diferenciaron significativamente en sus ganancias diarias de peso.

Se constató un comportamiento singular de los gazapos más livianos, que mostraban el menor aumento diario de peso en la semana previa a la separación de sus madres y en los que, contrariamente a lo esperado, el destete no afectó negativamente su tasa de crecimiento, sino que fue el grupo que presentó el mayor valor de la misma.

Las respuestas observadas en el crecimiento de los distintos grupos de gazapos ante el destete a los 25 días de vida indicarían que (a) los valores de ganancia diaria de peso de los gazapos del grupo liviano en la semana previa al destete serían atribuibles a un pobre ambiente de lactancia, ya sea por competencia con su hermano de camada o debido a un pobre desempeño de la madre, en cuyo caso el destete implica un mejoramiento de este ambiente; (b) los gazapos de los grupos intermedio y pesado muestran una disminución en su velocidad de crecimiento luego del destete, posiblemente debido al deterioro de su ambiente nutricional, pero una vez superado el estrés de la separación, no sólo recuperan el ritmo de ganancia de peso que tenían en la última semana de la lactancia, sino que incluso lo mejoran. 
La respuesta observada en los gazapos del grupo liviano resulta llamativa en tanto, durante la lactancia ellos tienen acceso al mismo tipo de alimento, tanto balanceado como voluminoso, que se les ofrece luego de la separación de su madre, por lo que puede especularse que en presencia de la hembra prefieren amamantarse antes que consumir alimento sólido, aún cuando la ingesta de leche sea insuficiente para asegurar un correcto crecimiento.

Al ser destetados, estos gazapos se ven obligados a consumir el alimento ofertado, hecho que se traduce en un mejor ambiente nutricional y, consecuentemente, repercute sobre su performance.

En base a estos resultados puede concluirse que, al igual que lo informado para el destete anticipado y el destete precoz ${ }^{9}$, el destete de los gazapos a los 25 días de vida es una práctica factible de ser aplicada en los criaderos comerciales de chinchilla en tanto la misma no repercute negativamente sobre el crecimiento posterior de las crías, a la vez que cumple con el objetivo de permitir que la hembra interrumpa su anestro lactacional y se reincorpore antes al ciclo reproductivo, contribuyendo de esta manera a disminuir el período inter-parto.

Agradecimientos. A los estudiantes de veterinaria de la Universidad Nacional de Rosario que participaron en el cuidado de los animales y en el registro de los datos.

\section{REFERENCIAS}

1. Aleandri F. 2000. Cría y comercialización de la chinchilla, Ed. Producciones Gráficas SRL, 2da. ed., Buenos Aires, $400 \mathrm{p}$.

2. Barabasz B, Lapinski S. 2008. Growth rate of sucking chinchilla pups and lactating performance of their dams. Anim Sci Pap Rep 26: 227-234.

3. Dzierżanowska D, Brzozowski M, Góral K. 2014. Young chinchillas weight gain, depending on their body mass at birth. Anim Sci 53: 95-101.

4. García A, García T. 2004. Chinchillas, Ed. Albatros, Buenos Aires, p. 81.

5. Grau J. 1986. La chinchilla. Su crianza en todos los climas, $3^{\mathrm{ra}} \mathrm{ed}$, El Ateneo, Buenos Aires, $213 \mathrm{p}$.

6. Kraft H. 1987. Diseases of chinchillas, Ed.TFH Publication Inc., Neptune City, USA, p. 49-52.

7. Neira R, García X, Scheu R. 1989. Análisis descriptivo del comportamiento reproductivo y de crecimiento de chinchillas en confinamiento. Avances Prod Anim 14: 109119.

8. Spotorno AE, Zuleta CA, Valladares JP, Deane, AL, Jiménez JE. 2004. Chinchilla laniger. Mammalian Species 758: $1-9$.

9. Weir BJ. 1970. Chinchilla. In: Reproduction and breeding techniques for laboratory animals (Hafez, E.S. Ed.), Lea \& Febiger, Philadelphia, p. 209-223.

\section{Revista Veterinaria ingresa a SciELO}

Scientific Electronic Library Online

Revista Veterinaria, publicación oficial de la Facultad de Ciencias Veterinarias de la Universidad Nacional del Nordeste (Corrientes, Argentina), ha logrado acceder al Núcleo Básico de Revistas Científicas Argentinas (Nivel 1), luego de calificar adecuadamente en el Centro Argentino de Información Científica y Tecnológica (CAICYT), según Resolución 2485/14 del Consejo Nacional de Investigaciones Científicas y Técnicas (CONICET).

Sobre un puntaje máximo de 33 se obtuvieron 32 puntos. Tal calificación constituye "una garantía de la excelencia de la publicación" (sic) y queda expedita la vía del Portal SciELO (Scientific Electronic Library Online) para los artículos publicados.

En tal calificación gravitó positivamente la circunstancia de haber aumentado el índice de impacto (Scimago-Elsevier) y haber disminuido las autocitaciones. También se tuvieron en cuenta aspectos como la amplia cobertura de la revista, la calidad científica del Comité Editorial, los criterios de evaluación de los artículos, el origen de los autores (locales 60\%, nacionales 13\%, extranjeros $27 \%$, en idioma inglés), el adecuado balance entre trabajos científicos originales y reseñas bibliográficas (ambos con alta calidad), así como el estricto cumplimiento de la periodicidad semestral y la favorable acogida por indizadores como Cab, J-Gate, Doaj, Ebsco, Gale Cengage, Infocyt, Latindex y Scopus.

Se consolida de esta manera la continuidad de "Revista Veterinaria", que en su acontecer registra más de 50 años de existencia en nuestra Facultad de Ciencias Veterinarias, entidad que en 2017 cumplió el $97^{\circ}$ aniversario de su fundación. 